\title{
Carcinoma de células de Merkel: revisão de literatura
}

\section{Merkel cell carcinoma: literature review}

Russian Soares Uchoa ${ }^{1}$. Felipe Ramos Nogueira ${ }^{1}$. Marcelo Leite Vieira Costa ${ }^{2,4}$. Annya Costa Araújo de Macedo Goes ${ }^{3,4}$.

1 Residência médica em Cirurgia Geral no Hospital Universitário Walter Cantídio, Fortaleza, Ceará, Brasil. 2 Doutorado em Farmacologia pela Universidade Federal do Ceará (UFC), Fortaleza, Ceará, Brasil. 3 Doutorado em Cirurgia pela Universidade Federal do Ceará (UFC), Fortaleza, Ceará, Brasil. 4 Professor(a) adjunto(a) da Disciplina de Cirurgia da Faculdade de Medicina da Universidade Federal do Ceará (UFC), Preceptor(a) da Residência de Cirurgia Geral do Hospital Universitário Walter Cantídio (HUWC), Fortaleza, Ceará, Brasil.

\section{RESUMO}

Carcinoma de células de Merkel (CCM) é uma neoplasia neuroendócrina cutânea rara e agressiva. A maioria dos casos ocorrem na região da cabeça e pescoço, em áreas intensamente expostas ao Sol, de pessoas idosas e brancas. CCM é letal em 33\% dos casos, demonstrando um prognóstico pior que o melanoma. Para melhorar o prognóstico dos pacientes com CCM deve ser realizado um diagnóstico precoce e o tratamento com adequado uso do esvaziamento linfonodal, da quimioterapia e radioterapia.

Palavras-chave: Carcinoma de célula de Merkel. Tratamento. Patogênese.

\section{ABSTRACT}

Merkel cell carcinoma (MCC) is a rare, aggressive neuroendocrine skin cancer. Most cases occur in the head and neck areas intensely exposed to the sun, of elderly and white people. MCC is lethal in 33\% of cases, demonstrating worse prognosis than melanoma. To improve the prognosis of patients with CCM should be performed early diagnosis and treatment with appropriate use of lymph node resection, chemotherapy and radiotherapy.

Keywords: Merkel cell carcinoma. Treatment. Pathogenesis.

Autor correspondente: Russian Soares Uchoa, Rua Senador Machado, 185, apartamento 1802, Mucuripe, Fortaleza, Ceará. CEP: 60165-170. Telefone: +55 85 99232-8484. E-mail: russian_uchoa@hotmail.com

Conflito de interesses: Não há qualquer conflito de interesses por parte de qualquer um dos autores.

Recebido em: 26 Mai 2016; Revisado em: 05 Dez 2016; Aceito em: 05 Dez 2016. 


\section{INTRODUÇÃO}

Carcinoma de células de Merkel (CCM) é uma neoplasia neuroendócrina cutânea rara e agressiva. Foi inicialmente descrita em 1972 por Toker como um câncer trabecular da derme com alto risco de metástase linfática, encontrado principalmente em pacientes da raça branca e idosos. ${ }^{1} \mathrm{~A}$ maioria dos casos ocorrem na região da cabeça e pescoço, em áreas intensamente expostas ao sol. CCM é letal em 33\% dos casos, demonstrando um prognóstico pior que o melanoma. ${ }^{2}$ Para melhorar o prognóstico dos pacientes com CCM deve ser realizado diagnóstico precoce e tratamento com adequado uso do esvaziamento linfonodal, quimioterapia e radioterapia. ${ }^{3}$

\section{EPIDEMIOLOGIA}

Com o advento de novos métodos de coloração imuno-histoquímica, tem aumentado os relatos de carcinoma de células de Merkel na última década. Cerca de 950 a 1300 novos casos de CCM são diagnosticados por ano nos EUA. Estes números vêm aumentando provavelmente devido ao avanço da idade da população, aumento da exposição a luz solar e ao aumento do número de indivíduos imunocomprometidos. ${ }^{4,5}$ Feng et al. descreveram recentemente um polyomavirus isolado a partir de amostras de CCM, o que pode justificar a maior incidência em pacientes imunocomprometidos. Os autores estudaram 10 casos e identificaram o vírus em 8 deles. Em pacientes que não eram portadores de CCM, foi identificado o vírus em $16 \%$ dos casos. ${ }^{6}$

A incidência anual de carcinoma de células de Merkel nos EUA é aproximadamente 0,23 para 100.000 indivíduos caucasianos. CCM acontece geralmente em pessoas idosas, com média de 69 anos ao diagnóstico. ${ }^{7,8}$ A incidência em pessoas do sexo masculino é maior (61\% do sexo masculino e $39 \%$ do sexo feminino). ${ }^{9}$ Outros tumores como o carcinoma de células escamosas e a leucemia linfocítica crônica podem estar associadas. $^{7}$

\section{ETIOLOGIA}

Ainda não se sabe uma etiologia específica clara para o CCM, porém prováveis fatores de risco têm sido observados em séries de casos e estudos de base populacional. A história de exposição ao sol ou a concordância de outras condições de pele associada ao sol e o carcinoma de células escamosas em particular é comum em pacientes com CCM. ${ }^{10,11}$ Estudos também têm demonstrado um aumento da incidência de CCM em pacientes em uso de imunossupressores para transplante de órgãos sólidos, ${ }^{12}$ em pacientes com infecção pelo Vírus da Imunodeficiência Humana (HIV) e baixas contagens de CD $4,{ }^{13}$ em pacientes submetidos à radioterapia ou quimioterapia ${ }^{14,15} \mathrm{e}$ em pacientes cronicamente expostos ao arsênio.

A mais recente descoberta sobre a patogênese do CCM foi a descoberta de um novo polyomavírus denominado polyomavirus da célula de Merkel (VCM) por Moore e Chang. ${ }^{6}$ Feng et al. ${ }^{6}$ descobriram e sequenciaram o genoma do VCM identificando DNA do polyomavírus em 8 de 10 tumores CCM. Desde o relatório original, uma multidão de outros grupos têm encontrado o DNA do polyomavírus em amostras de tecido do CCM. Embora a pesquisa do DNA do VCM possa ser útil na distinção entre o CCM de outras neoplasias cutâneas e na identificação do tumor primário na doença metastática, Tolstov et al. ${ }^{16}$ relatam $50 \%$ e $80 \%$ de prevalência de anticorpos contra o polyomavírus entre as pessoas menores de 15 anos e acima de 50 anos, respectivamente.

\section{APRESENTAÇÃO CLÍNICA}

As lesões do CCM têm sido descritas na literatura como uma massa subcutânea classicamente indolor com uma aparência cística ou nodular, mas por vezes com uma aparência semelhante a placa, que pode ser rodeado por pequenas lesões satélites. O tamanho do tumor varia de 2-200 mm, mas é mais frequentemente $<20 \mathrm{~mm} \cdot{ }^{17}$ As lesões podem variar na cor, na maioria das vezes encontradas como vermelho/rosa, azul/violáceas ou da cor da pele. ${ }^{17}$ Eles podem apresentar telangiectasia sobreposta ou uma superfície brilhante, fazendo lesões facilmente confundidas com carcinoma basocelular. ${ }^{18}$ Devido a sua aparência clínica variável e inespecífica, o CCM raramente é suspeito antes da biopsia.

Os tumores podem apresentar um crescimento rápido, exigindo um rápido diagnóstico e início do tratamento. ${ }^{19} \mathrm{~A}$ região da cabeça e pescoço é citada como a topografia de maior incidência para CCM, aproximadamente $50 \%$ dos casos, seguido em ordem decrescente de extremidades, tronco e nádega. ${ }^{19}$

Estudos evidenciaram que $71-79 \%$ dos pacientes apresentam envolvimento linfonodal clinicamente negativo, $19-24 \%$ apresentam metástase linfonodal regionais, e 5\% têm evidência de doença metastática a distância. ${ }^{20}$ Os principais sítios de lesão secundária são: pele (28\%), nódulos linfáticos (27\%), fígado (13\%), pulmão $(10 \%)$, osso $(10 \%)$ e cérebro $(6 \%) .{ }^{18,19}$

\section{PROPEDÊUTICA}

A análise inicial deve consistir em avaliação clínica do sitio primário da lesão com observação de lesões satélites. Hemograma completo, eletrólitos séricos e fosfatase alcalina são úteis. ${ }^{18}$

Os pacientes com doença localizada, sem evidência física ou sintomas de metástases regionais ou distantes, devem ser avaliados se houve disseminação para cadeias linfonodais regionais com biópsia de linfonodo sentinela ou ultrassonografia. ${ }^{21}$ Os pacientes com lesões suspeitas em linfonodos regionais deverão ser submetidos a Punção Aspirativa por Agulha Fina (PAAF), muitas vezes, guiada por ultrassonografia para a amostragem mais precisa. Quando os nódulos linfáticos são positivos, a avaliação radiológica de corpo inteiro é geralmente indicada para detectar metástases distantes. ${ }^{21}$ Essa avaliação pode ser realizada com a tomografia por emissão de pósitrons (PET-TC). ${ }^{22}$ Em um estudo recente com 18 pacientes, o estadiamento foi ajustado em 33\% dos pacientes, e a abordagem de tratamento foi alterada em $43 \%$ dos pacientes após resultados do PET-TC. ${ }^{23}$ Tomografia 
computadorizada (TC) de alta resolução e Ressonância magnética (RM) podem ser usados em circunstâncias especiais. $^{21} \mathrm{TC}$ é útil para a imagem latente do tórax e do abdômen para excluir lesões metastáticas. Ressonância Magnética tem sido recomendado para avaliação local e de linfonodos regionais para pacientes com CCM e, em especial, para avaliação da disseminação local do tumor em regiões de difícil acesso por ultrassonografia e regiões mais profundas da região de cabeça e pescoço, tais como a região sinusal e órbita.

\section{BIÓPSIA}

A punção aspirativa por agulha fina (PAAF) permite um diagnóstico não invasivo e precoce deste tumor agressivo para facilitar o planejamento inicial do tratamento, podendo ser facilmente realizada em pacientes idosos em comparação com biópsia excisional. As características citológicas em aspirado incluem o aumento da celularidade, grupos sem coesão de células malignas de tamanho pequeno a médio, com núcleos uniformes e arredondados com efeito de moldagem, cromatina fina, múltiplos micronucléolos e citoplasma escasso. ${ }^{24}$ Uma vez que este tumor tem um potencial altamente maligno para recorrência local, disseminação linfonodal e distante, e muitas vezes é combinado com outros tumores, é importante realizar PAAF ou biópsia de lesões diferentes em um mesmo paciente. O diagnóstico definitivo é feito por análises histológicas, especialmente métodos de imunohistoquímica (detecção de filamentos intermediários e marcadores neuroendócrinos). ${ }^{25}$

Histologicamente, CCM surge na derme e se estende para o tecido subcutâneo. A epiderme é raramente envolvida e a pele sobrejacente é raramente ulcerada. O tumor pode se consistir em células isoladas, coesas e estruturas como rosetas.

Os três principais padrões histológicos são:

1 - tipo sólido - mais comum, composto por grupos irregulares de células tumorais interligadas por fios de tecido conjuntivo;

2 - tipo trabecular - cordões de células bem definidas que formam colunas ou cabos;

3 - tipo difuso - apresenta fraca coesão e um tipo de crescimento como linfoma difuso.

A coexpressão globular paranuclear de citoqueratina e neurofilamentos por um tumor dérmico indiferenciado é de grande ajuda no diagnóstico de CCM e ajuda a diferenciá-lo do carcinoma de pequenas células. ${ }^{26}$ A microscopia eletrônica demonstra as características patognomônicas para este tumor: grânulos neurosecretórios de corpo denso, com diâmetro de 100-250nm cercado por espirais de filamentos intermediários. Sob o microscópio eletrônico de varredura, numerosos processos semelhantes a dedos, tinham sido descritos por Yamashita et al. ${ }^{27}$ Resultados imunohistoquímicos são universalmente positivos para citoqueratina.

Estudos recentes demonstram anormalidades cromossômicas em cromossomos 1, 11 e $13 .^{28}$ Análise de hibridização genômica comparativa revelou um padrão de ganhos e perdas que se assemelha ao observado no câncer de pulmão de pequenas células. Perdas foram observados para cromossomas $3 p(46 \%), 5 q(21 \%), 8 p(21 \%), 10$ (33\%), 11q (17\%), 13q (33\%) e $17 \mathrm{p}$ (25\%). Ganhos significativos foram observados para os cromossomos 1 (63\%), 3T (33\%), 5p (38\%), 8q (38\%), $19(63 \%)$ e X (41\%), com números menores tendo ganhos para os cromossomos 6, 7, 20 e 21 .

Armadilhas de diagnóstico incluem o seguinte:

- a coexistência de CCM em associação com carcinoma espinocelular e basocelular. ${ }^{29}$ Isto tem a implicação de que um carcinoma de células escamosas ou basocelular deve ser seccionado cuidadosamente para evitar a falta do componente do CCM;

- presença de desmoplasia pode mascarar o diagnóstico de CCM;

- linfoma é um importante diagnóstico diferencial de tumores cutâneos de células redondas e azuis. A imunohistoquímica e, se necessário, reação em cadeia da polimerase e sequenciamento são ferramentas úteis para diferenciá-los.

A Johns Hopkins School of Medicine realizou a aplicação de técnicas moleculares para detecção de vírus de células de Merkel em Linfonodo sentinela. Oito em cada 25 (32\%) amostras apresentaram vírus detectável sem doença microscópica. Isto pode identificar um subgrupo de pacientes que se beneficiariam de tratamento adjuvante linfonodal.

\section{CLASSIFICAÇÃO}

Yiengpruksawan ${ }^{30}$ propôs uma classificação para CCM da seguinte forma: Estágio I seria restrita à pele, Estágio II incluiria metástase linfonodal e Estágio III seria caracterizado por metástases à distância. Mais tarde, a doença de Estágio I foi classificada como IA $(\leq 2 \mathrm{~cm})$ ou IB $(>2 \mathrm{~cm})$ com base em publicações de Yiengpruksawan e Allen..$^{30,31}$ No entanto, esse estudo não foi capaz de mostrar diferenças estatísticas no prognóstico entre grupos de tamanhos diferentes do tumor. Num estudo de 251 pacientes, Allen et al. mostraram que o sistema de estadiamento proposto com a divisão de IA e IB resultou num prognóstico diferente; assim, eles usaram um sistema de quatro estágios ${ }^{31}$ definidos da seguinte forma:

- $\quad$ Estágio I: $<2 \mathrm{~cm}$.

- $\quad$ Estágio II: $\geq 2 \mathrm{~cm}$, sem evidência de doença regional ou distante.

- Estágio III: com linfonodos positivos na cadeia de drenagem linfonodal.

- $\quad$ Estágio IV: com metástases à distância.

Após uma média de quarenta meses de seguimento, as taxas de sobrevivência foram de $81 \%$ para o Estágio I, 
$67 \%$ para o Estágio II, de 52\% para o Estágio III e 11\% para o Estágio IV. ${ }^{32}$

A sétima edição do American Joint Committee on Cancer $(\mathrm{AJCC})^{33}$ trouxe uma nova classificação conforme definido abaixo:

- $\quad$ Tumor primário (T):

○ TX: o tumor primário não pode ser avaliado.

○ T0: não há evidência de tumor primário.

○ Tis: tumor primário in situ.

○ T1: dimensão máxima do tumor $\leq 2 \mathrm{~cm}$

- T2: dimensão máxima do tumor $>2 \mathrm{~cm}$, mas $\leq 5 \mathrm{~cm}$.

○ T3: dimensão máxima do tumor $>5 \mathrm{~cm}$.

- T4: o tumor primário invade ossos, músculos, fáscias ou cartilagem.

- $\quad$ Linfonodos Regionais (N):

○ NX: linfonodo regional não pode ser avaliado.

○ N0: sem metástases em linfonodos regionais.

- cN0: linfonodos negativos ao exame clínico.

- pN0: linfonodos negativos ao exame patológico.

○ N1: metástases em linfonodos regionais.

- N1A: micrometástase.

- N1B:. macrometástase.

○ N2: metástase em trânsito.

- $\quad$ Metástase a distância (M):

○ M0: sem metástases à distância.

○ M1: metástases além de linfonodos regionais:

- M1a: metástases para a pele, tecidos subcutâneos, ou linfonodos distantes.

- M1b: metástase de pulmão.

- M1c: metástases para todos os outros sítios viscerais.

\section{TRATAMENTO}

O tratamento de escolha para o CCM depende de muitos fatores, incluindo a fase do tumor, o envolvimento linfonodal, a localização do tumor e as comorbidades médicas do paciente. Embora longe de um consenso, a maioria das recomendações atuais incluem a adição de radioterapia (RT) adjuvante para reduzir recidiva local ou linfonodal e melhorar a sobrevida da doença.

\section{Cirurgia}

O padrão de tratamento cirúrgico para o CCM tem sido tradicionalmente uma ampla excisão local. Alguns especialistas sugeriram que a cirurgia por si só é adequada para a doença em estágio inicial. ${ }^{32-34}$ Um estudo recente de 95 pacientes, a partir de um banco de dados do Instituto Nacional do Câncer em Milão, que avaliou o uso isolado da cirurgia nas fases iniciais do CCM evidenciou uma taxa de $80 \%$ de sobrevida em 5 anos quando os linfonodos eram patologicamente negativos, que caiu para $58 \%$ quando metástases regionais estavam presentes. ${ }^{35}$ Embora tenha sido inicialmente pensado que as margens $\geq 3 \mathrm{~cm}$ eram necessárias para a melhor redução de recorrência local, ${ }^{36,37}$ margens de $2-3 \mathrm{~cm}$ de largura e $2 \mathrm{~cm}$ de profundidade são agora aceitas. $^{38,39}$

Tal como acontece com os cânceres de pele mais comuns. A microcirurgia de Mohs é uma opção atraente para o tratamento de tumores na cabeça e pescoço, onde a largura da margem recomendada e profundidade são ou esteticamente indesejável ou tecnicamente impossível. Boyer et al. avaliaram as taxas de recorrência e sobrevida em pacientes com estágio I do CCM tratados com cirurgia de Mohs, com ou sem RT adjuvante e concluiram que a adição de RT adjuvante após a cirurgia de Mohs não era necessário, uma vez que não forneceu benefício adicional em termos de recorrência ou sobrevivência. ${ }^{40}$ O'Connor et al. avaliaram os doentes tratados com ampla excisão local ou cirurgia de Mohs e evidenciaram que, enquanto a excisão cirúrgica padrão resultou em persistência local e metástases regionais em $31,7 \%$ e 48,8\% dos doentes respectivamente, a cirurgia de Mohs resultou em apenas $6,8 \%$ e $33,3 \%$ persistência local e metástases regionais, respectivamente. ${ }^{41} \mathrm{O}^{\prime}$ Connor et al sugeriram que pode haver alguma vantagem em adicionar RT adjuvante após a cirurgia de Mohs para reduzir ainda mais o risco de metástases linfonodais.

Outro ponto de discordância entre os cirurgiões é a realização ou não da biopsia de linfonodo sentinela para pacientes submetidos à excisão cirúrgica do CCM. Mesmo em pacientes sem evidências clínicas de envolvimento de gânglios linfáticos, $25 \%$ a 100\% dos linfonodos de drenagem primária podem conter metástases microscópicas, ${ }^{42}$ que podem levar à recidiva linfonodal em até $76 \%$ dos pacientes. Allen et al. mostraram dados sugerindo que incidência de linfonodos positivos foi independente do tamanho do tumor, de modo que a biopsia de linfonodo sentinela de rotina deve ser realizada em todos os pacientes submetidos à excisão cirúrgica do CCM. ${ }^{33}$

\section{Radioterapia}

A radioterapia (RT) tem sido defendida por alguns autores como tratamento definitivo, especialmente para os pacientes que são pobres candidatos à cirurgia. $^{43,44}$ Ainda que os 
números sejam pequenos, estudos mostram que a RT pode ser uma alternativa viável para pacientes incapazes de suportar uma cirurgia. ${ }^{43,44}$

A maioria dos estudos retrospectivos realizados ao longo dos últimos 15 anos têm demonstrado consistentemente melhor controle local após a cirurgia quando RT adjuvante é adicionada. ${ }^{45-47}$ Duas metanálises foram realizadas com o objetivo de avaliar comparativamente o tratamento isolado com cirurgia e o tratamento com cirurgia + RT, sendo observado redução da recidiva e aumento da sobrevida nos pacientes que foram submetidos ao tratamento combinado de cirurgia + RT. ${ }^{45,46}$ Embora esses estudos não sejam substitutos para um ensaio clínico randomizado prospectivo, a maioria dos especialistas parecem concordar que RT adjuvante beneficia pacientes em termos de diminuição da recorrência e sobrevida global. As diretrizes recentemente atualizadas pelo National Comprehensive Cancer Network recomendam o uso rotineiro de RT adjuvante para os nódulos linfáticos em todos os casos, a menos que o paciente tenha uma biopsia de linfonodo sentinela negativa. ${ }^{47}$

\section{REFERÊNCIAS}

1. Toker C. Trabecular carcinoma of the skin. Arch Dermatol. 1972;105(1):107-10.

2. Hodgson NC. Merkel cell carcinoma: changing incidence trends. J Surg Oncol. 2005;89(1):1-4.

3. Kim JA, Choi AH. Effect of radiation therapy on survival in patients with resected Merkel cell carcinoma: a propensity score surveillance, epidemiology, and end results database analysis. JAMA Dermatol. 2013;149(7):831-8.

4. Eng TY, Boersma MG, Fuller CD, Goytia V, Jones WE 3rd, Joyner $\mathrm{M}$, et al. A comprehensive review of the treatment of Merkel cell carcinoma. Am J Clin Oncol. 2007;30(6):624-36.

5. Agelli M, Clegg LX. Epidemiology of primary Merkel cell carcinoma in the United States. J Am Acad Dermatol. 2003;49(5):832-41.

6. Feng H, Shuda M, Chang Y, Moore PS. Clonal integration of a polyomavirus in human Merkel cell carcinoma. Science. 2008;319(5866):1096-1100.

7. Kaae J, Hansen AV, Biggar RJ, Boyd HA, Moore PS, Wohlfahrt J, et al. Merkel cell carcinoma: incidence, mortality, and risk of other cancers. J Natl Cancer Inst. 2010;102(11):793-801.

8. Pectasides D, Pectasides M, Economopoulos T. Merkel cell cancer of the skin. Ann Oncol. 2006;17(10):1489-95.

9. Lemos BD, Storer BE, Iyer JG, Phillips JL, Bichakjian CK, Fang LC, et al. Pathologic nodal evaluation improves prognostic accuracy in Merkel cell carcinoma: analysis of 5823 cases as the basis of the first consensus staging system. J Am Acad Dermatol. 2010;63(5):751-61.

10. Cerroni L, Kerl H. Primary cutaneous neuroendocrine (Merkel cell) carcinoma in association with squamous- and basal-cell carcinoma. Am J Dermatopathol. 1997;19(6):610-13.

11. Popp S, Waltering S, Herbst C, Moll I, Boukamp P. UV-B-type mutations and chromosomal imbalances indicate common pathways

\section{Quimioterapia}

Devido à semelhança entre os tumores, a maioria dos regimes de quimioterapia utilizados para tratar o CCM são similares aos encontrados para o tratamento do câncer de pulmão de pequenas células. A carboplatina, cisplatina, etoposido, ciclofosfamida, 5-fluorouracil, doxorrubicina e vincristina são alguns dos agentes mais comumente utilizados. Tradicionalmente, a quimioterapia primária foi reservada para aqueles pacientes com doença irressecável, quer devido à má candidatura cirúrgica por causa de doença metastática ou devido à presença de comorbidades médicas. A quimioterapia adjuvante tem sido sugerida para ser benéfica quando adicionado a cirurgia e RT adjuvante para o tratamento da doença no estágio II. ${ }^{18}$ Allen et al. ${ }^{33}$ não relataram nenhum benefício na sobrevivência após a adição de quimioterapia adjuvante. Por outro lado, vários autores têm relatado que a quimioterapia pode ser benéfica em termos de recorrência e sobrevivência, especialmente para pacientes com elevado risco de CCM metastático.

for the development of Merkel and skin squamous cell carcinomas. Int J Cancer. 2002;99:352-60.

12. Penn I, First MR. Merkel's cell carcinoma in organ recipients: report of 41 cases. Transplantation. 1999;68(11):1717-21.

13. Engels EA, Frisch M, Goedert JJ, Biggar RJ, Miller RW. Merkel cell carcinoma and HIV infection. Lancet. 2002;359(9305):497-8.

14. Tuneu A, Pujol RM, Moreno A, Barnadas MA, de Moragas JM. Postirradiation Merkel cell carcinoma. J Am Acad Dermatol. 1989;20(3):505-7.

15. Lunder EJ, Stern RS. Merkel-cell carcinomas in patients treated with methoxsalen and ultraviolet A radiation. N Engl J Med. 1998;339(17):1247-8.

16. Tolstov YL, Pastrana DV, Feng H, Becker JC, Jenkins FJ, Moschos $\mathrm{S}$, et al. Human Merkel cell polyomavirus infection II. MCV is a common human infection that can be detected by conformational capsid epitope immunoassays. Int J Cancer. 2009;125(6):1250-6.

17. Heath M, Jaimes N, Lemos B, Mostaghimi A, Wang LC, Peñas PF, et al. Clinical characteristics of Merkel cell carcinoma at diagnosis in 195 patients: the AEIOU features. J Am Acad Dermatol. 2008;58(3):375-81.

18. Poulsen M. Merkel cell carcinoma of skin: diagnosis and management strategies. Drugs Aging. 2005;22(3):219-29.

19. Goessling W, McKee PH, Mayer RJ. Merkel cell carcinoma. J Clin Oncol. 2002;20(2):588-98.

20. Stokes JB, Graw KS, Dengel LT, Swenson BR, Bauer TW, Slingluff CL Júnior, et al. Patients with Merkel cell carcinoma tumors $\leq 1.0 \mathrm{~cm}$ in diameter are unlikely to harbor regional lymph node metastasis. J Clin Oncol. 2009;27(23):3772-7.

21.Zager JS, Brodsky S, Berman CG. Imaging of Merkel cell carcinoma. Curr Probl Cancer. 2010;34(1):65-76. 
22. Peloschek P, Novotny C, Mueller-Mang C, Weber M, Sailer $\mathrm{J}$, Dawid M, et al. Diagnostic imaging in Merkel cell carcinoma: lessons to learn from 16 cases with correlation of sonography, CT, MRI and PET. Eur J Radiol. 2010;73(2):317-23.

23. Concannon R, Larcos GS, Veness M. The impact of (18)F-FDG PET-CT scanning for staging and management of Merkel cell carcinoma: results from Westmead Hospital, Sydney, Australia. J Am Acad Dermatol. 2010;62(1):76-84.

24. Ostović KT, Haris V, Miletić Z, Lambasa S, Lajtman Z, StoosVeić T. Fine needle aspiration cytology of metastatic Merkel cell carcinoma. Coll Antropol. 2010;34(2):691-6.

25. Meyer-Pannwitt U, Kummerfeldt K, Boubaris P, Caselitz J. Merkel cell carcinoma. Langenbecks Archiv fur Chirurgie. 1997;382(6):349-58.

26. Ratner D, Nelson BR, Brown MD, Johnson TM. Merkel cell carcinoma. J Am Acad Dermatol. 1993;29(2 Pt I):143-60.

27. Yamashita Y, Toida K, Ogawa H. Observation of Merkel cells with scanning electron microscopy. Neurosci Lett. 1993;159(12):155-8.

28. Leonard JH, Leonard P, Kearsley JH. Chromosomes 1, 11, and 13 are frequently involved in karyotypic abnormalities in metastatic Merkel cell carcinoma. Cancer Genet Cytogenet. 1993;67(1):65-70.

29. Cerroni L, Kerl H. Primary cutaneous neuroendocrine (Merkel cell) carcinoma in association with squamous- and basal-cell carcinoma. Am J Dermatopathol. 1997;19(6):610-13.

30. Yiengpruksawan A, Coit DG, Thaler HT, Urmacher C, Knapper WK. Merkel cell carcinoma. Prognosis and management. Arch Surg. 1991;126(12):1514-9.

31.Eng TY, Boersma MG, Fuller CD, Cavanaugh SX, Valenzuela F, Herman TS. Treatment of merkel cell carcinoma. Am J Clin Oncol. 2004;27(5):510-5.

32. Allen PJ, Bowne WB, Jaques DP, Brennan MF, Busam K, Coit DG. Merkel cell carcinoma: prognosis and treatment of patients from a single institution. J Clin Oncol. 2005;23(10):2300-9.

33. Edge SB, Byrd DR, Compton CC. AJCC cancer staging manual. 7. Ed. Chicago: Springer; 2010. Chapter 30, Merkel Cell Carcinoma; p. 315-9.

34. Mann GB, Allen PJ, Coit DG. Merkel cell carcinoma. Aust N Z J Surg. 1999;69:87.
35. Bajetta E, Celio L, Platania M, Lo Vullo S, Patuzzo R, Maurichi A, et al. Single-institution series of early-stage Merkel cell carcinoma: long-term outcomes in 95 patients managed with surgery alone. Ann Surg Oncol. 2009;16(11):2985-93.

36. O'Connor WJ, Brodland DG. Merkel cell carcinoma. Dermatol Surg. 1996;22(3):262-7.

37. Fenig E, Brenner B, Katz A, Rakovsky E, Hana MB, Sulkes A. The role of radiation therapy and chemotherapy in the treatment of Merkel cell carcinoma. Cancer. 1997;80(5):881-5.

38. Lawenda BD, Thiringer JK, Foss RD, Johnstone PA. Merkel cell carcinoma arising in the head and neck: optimizing therapy. Am J Clin Oncol. 2001;24(1):35-42.

39. Veness MJ. Merkel cell carcinoma (primary cutaneous neuroendocrine carcinoma): an overview on management. Australas J Dermatol. 2006;47(3):160-5.

40. Boyer JD, Zitelli JA, Brodland DG, D’Angelo G. Local control of primary Merkel cell carcinoma: review of 45 cases treated with Mohs micrographic surgery with and without adjuvant radiation. J Am Acad Dermatol. 2002;47(6):885-92.

41. O'Connor WJ, Roenigk RK, Brodland DG. Merkel cell carcinoma. Comparison of Mohs micrographic surgery and wide excision in eighty-six patients. Dermatol Surg. 1997;23(10):929-33.

42.Allen PJ, Zhang ZF, Coit DG. Surgical management of Merkel cell carcinoma. Ann Surg. 1999;229(1):97-105.

43. Mortier L, Mirabel X, Fournier C, Piette F, Lartigau E. Radiotherapy alone for primary Merkel cell carcinoma. Arch Dermatol. 2003;139(12):1587-90.

44. Veness M, Foote M, Gebski V, Poulsen M. The role of radiotherapy alone in patients with Merkel cell carcinoma: reporting the Australian experience of 43 Patients. Int J Radiat Oncol Biol Phys. 2010;78(3):703-9.

45. Lewis KG, Weinstock MA, Weaver AL, Otley CC. Adjuvant local irradiation for Merkel cell carcinoma. Arch Dermatol. 2006;142(6):693-700.

46. Mojica P, Smith D, Ellenhorn JD. Adjuvant radiation therapy is associated with improved survival in Merkel cell carcinoma of the skin. J Clin Oncol. 2007;25(9):1043-47.

47. Engstrom PF, Arnoletti JP, Benson AB 3rd, Chen YJ, Choti MA, Cooper HS, et al. NCCN Clinical Practice Guidelines in Oncology. J Natl Compr Canc Netw. 2009;7(8):778-831.

\section{Como citar:}

Uchoa RS, Nogueira FR, Costa ML, Goes AC. Carcinoma de células de Merkel: revisão de literatura. Rev Med UFC. 2017 mai-ago;57(2):44-49. 